\title{
ARTICLE \\ A distributed frontotemporal network underlies gamma-band synchronization impairments in schizophrenia patients
}

\author{
Daisuke Koshiyama ${ }^{1}$, Makoto Miyakoshi ${ }^{2}$, Yash B. Joshi ${ }^{1,3}$, Juan L. Molina ${ }^{1}$, Kumiko Tanaka-Koshiyama ${ }^{1}$, Joyce Sprock ${ }^{1}$, David L. Braff ${ }^{1}$,
} Neal R. Swerdlow iD ${ }^{1}$ and Gregory A. Light ${ }^{1,3}$

Synaptic interactions between parvalbumin-positive $\gamma$-aminobutyric acid (GABA)-ergic interneurons and pyramidal neurons evoke cortical gamma oscillations, which are known to be abnormal in schizophrenia. These cortical gamma oscillations can be indexed by the gamma-band auditory steady-state response (ASSR), a robust electroencephalographic (EEG) biomarker that is increasingly used to advance the development of novel therapeutics for schizophrenia, and other related brain disorders. Despite promise of ASSR, the neural substrates of ASSR have not yet been characterized. This study investigated the sources underlying ASSR in healthy subjects and schizophrenia patients. In this study, a novel method for noninvasively characterizing source locations was developed and applied to EEG recordings obtained from 293 healthy subjects and 427 schizophrenia patients who underwent ASSR testing. Results revealed a distributed network of temporal and frontal sources in both healthy subjects and schizophrenia patients. In both groups, primary contributing ASSR sources were identified in the right superior temporal cortex and the orbitofrontal cortex. In conjunction with normal activity in these areas, schizophrenia patients showed significantly reduced source dipole density of gamma-band ASSR (ITC > 0.25) in the left superior temporal cortex, orbitofrontal cortex, and left superior frontal cortex. In conclusion, a distributed network of temporal and frontal brain regions supports gamma phase synchronization. We demonstrated that failure to mount a coherent physiologic response to simple $40-\mathrm{Hz}$ stimulation reflects disorganized network function in schizophrenia patients. Future translational studies are needed to more fully understand the neural mechanisms underlying gamma-band ASSR network abnormalities in schizophrenia.

Neuropsychopharmacology (2020) 45:2198-2206; https://doi.org/10.1038/s41386-020-00806-5

\section{INTRODUCTION}

The gamma-band auditory steady-state response (ASSR) is a robust biomarker that is increasingly studied in neuropsychiatric disorders [1, 2]. Galambos et al. [3, 4] and Makeig [5] first demonstrated that gamma oscillations measured by scalp electroencephalography (EEG) recordings in response to $40-\mathrm{Hz}$ stimulation represent a preferred resonance frequency to investigate auditory information processing in humans; smaller responses were observed in response to lower or faster rates of stimulation. Kwon et al. [6] performed the first study of 40-Hz ASSR in schizophrenia patients using EEG and found selectively reduced power and synchronization in response to $40-\mathrm{Hz}$ stimulation. This initial finding has been replicated and extended in many subsequent studies using EEG [7-16] or magnetoencephalography (MEG) [17-21], including findings relating ASSR dysfunction to important domains of cognitive and psychosocial functioning in schizophrenia patients $[11,13,14]$. A recent meta-analysis confirmed that the $40-\mathrm{Hz}$ ASSR is a robust index of gamma synchronization deficits in schizophrenia patients [22]. Altered 40$\mathrm{Hz}$ ASSR has also been reported in patients with neuropsychiatric disorders other than schizophrenia, including bipolar disorder $[12,23-27]$, autism spectrum disorder [21, 28], as well as $22 q 11.2$ deletion syndrome [29].
Synaptic interactions between parvalbumin-positive $\gamma$-aminobutyric acid (GABA)-ergic interneurons and pyramidal neurons evoke cortical gamma oscillations [30, 31]. Abnormalities in parvalbumin-positive GABAergic interneurons, such as reduced expression of the GABA-synthesizing enzyme glutamic acid decarboxylase 67 (GAD67) [32] and parvalbumin [33] in cortical interneurons have been observed in postmortem brains of individuals with schizophrenia. In parallel, animal models of schizophrenia suggest that altered N-methyl-D-aspartate (NMDA) receptor signaling onto parvalbumin-positive interneurons results in gamma oscillation deficits in cortical microcircuits $[34,35]$. Thus, a clearer mechanistic explanation of how gamma oscillations are evoked in response to steady-state stimulation has already advanced our understanding of GABAergic interneuron dysfunction in schizophrenia [36, 37] and improved the potential use of ASSR biomarkers in the development of novel therapeutics for central nervous system disorders [38]. Still, crucial knowledge gaps exist in fully understanding ASSR and its implications for the pathogenesis of neuropsychiatric disorders.

Despite interest in the use of ASSR as a translational biomarker, there is limited information about the primary sources that underlie ASSR generation [39, 40]. Tada et al. [40] investigated the temporal response dynamics of gamma-band ASSR across

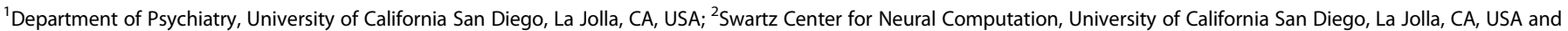
${ }^{3}$ VISN-22 Mental Illness, Research, Education and Clinical Center (MIRECC), VA San Diego Healthcare System, San Diego, CA, USA
}

Correspondence: Makoto Miyakoshi (mmiyakoshi@ucsd.edu)

Received: 25 April 2020 Revised: 2 August 2020 Accepted: 7 August 2020

Published online: 22 August 2020 
spatially distributed cortical surfaces in humans using electrocorticography (ECoG). They demonstrated prominent increases of gamma oscillations in primary auditory cortex (A1) and sensorimotor cortex as well as moderate increases of activity at prefrontal gyrus. Although the findings from Tada et al. [40] were from patients undergoing neurosurgical interventions for treatment refractory epilepsy, they nevertheless confirmed that these locations (primary auditory cortex, sensorimotor cortex, and prefrontal gyrus) are critical generators of ASSR in humans [40]. The loci underlying ASSR abnormalities in schizophrenia and their contributions to deficits in the generation of evoked gamma-band oscillatory activity remains unclear.

To address the issue of loci that underlie ASSR generation, a novel computational approach using independent components analysis (ICA) was developed to maximally leverage spatiotemporal relationships contained in multichannel scalp EEG recordings [41, 42]. ICA modeling facilitates the functional segregation of the multiple source interactions underlying ASSR generation in both temporal and spatial domains. In the current study, we applied a noninvasive blind-source separation approach to EEG source modeling in order to characterize the neural substrates underlying normal and impaired gamma-band ASSR in healthy subjects and schizophrenia patients. In addition, to test effects of medication, we applied the same analysis to the subset of patients who were not prescribed antipsychotics. Furthermore, we performed exploratory analyses to show correlations of gammaband ASSR at each estimated location with clinical symptoms, neurocognitive function, and functional outcomes in schizophrenia patients.

\section{MATERIALS AND METHODS}

Subjects

Participants included 293 healthy comparison subjects and 427 schizophrenia patients (Supplementary Table 1). Healthy comparison subjects were recruited through internet advertisements. Patients were recruited from community residential facilities and via clinician referral, and diagnosed using a modified version of the Structured Clinical Interview for DSM-IV-TR (SCID). Antipsychotic medications were prescribed for 389 schizophrenia patients and were not for 35 patients ( 3 patients did not provide antipsychotic medication data). Exclusion criteria is shown in Supplementary Method 1. Written informed consent was obtained from each subject. Audiometric testing was used to ensure that all participants could detect $1000-\mathrm{Hz}$ tones at $40 \mathrm{~dB}$ and to exclude mild hearing losses [43]. The Institutional Review Board of University of California San Diego approved all experimental procedures $(071128,071831,170147)$. Details of assessment of cognition, clinical symptoms and functional outcomes are shown in Supplementary Method 2.
Stimuli and procedures

Auditory steady-state stimuli were presented to subjects by means of foam insert earphones (Model 3A; Aearo Company Auditory Systems, Indianapolis, Indiana). The stimuli were 1-millisecond, 93dB clicks presented at $40 \mathrm{~Hz}$ in 500 -millisecond trains (Fig. 1). A block typically contained 200 trains of the clicks with 500-ms intervals. During the session, participants watched a silent cartoon video.

Electroencephalography recording and preprocessing

EEG data were continuously digitized at a rate of $1000 \mathrm{~Hz}$ (nose reference, forehead ground) using a 40-channel Neuroscan system (Neuroscan Laboratories, El Paso, Texas). The electrode montage was based on standard positions in the International 10-5 electrode system [44] fit to the Montreal Neurological Institute (MNI) template head Collins27 used in EEGLAB [45], including AFp10 and AFp9 as horizontal EOG electrodes, IEOG and SEOG above and below the left eye as vertical EOG electrodes, Fp1, Fp2, F7, F8, Fz, F3, F4, FC1, FC2, FC5, FC6, C3, Cz, C4, CP1, CP2, CP5, CP6, P7, P3, Pz, P4, P8, T7, T8, TP9, TP10, FT9, FT10, PO9, PO10, O1, O2, and Iz. Electrode-to-skin impedance mediated by conductive gel was brought below $4 \mathrm{k} \Omega$. The system acquisition band pass was 0.5-100 Hz. Offline, EEG data were imported to EEGLAB 14.1.2 [46] running under Matlab 2017b (The MathWorks, Natick, MA). Data were high-pass filtered [finite impulse response (FIR), Hamming window, cutoff frequency $0.5 \mathrm{~Hz}$, transition bandwidth 0.5$]$. EEGLAB plugin clean_rawdata() including Artifact Subspace Reconstruction (ASR) was applied to reduce high-amplitude artifacts [47-52]. The parameters used were: flat line removal, $10 \mathrm{~s}$; electrode correlation, 0.7; ASR, 20; window rejection, 0.5 . Mean channel rejection rate was $4.2 \%$ (SD 2.3, range 0-15.8). Mean data rejection rate was $2.0 \%$ (SD 3.5, range $0-22.4$ ). The rejected channels were interpolated using EEGLAB's spline interpolation function. Data were re-referenced to average. Adaptive mixture ICA $[41,53,54]$ was applied to the preprocessed scalp recording data to obtain temporally maximally independent components (ICs). For scalp topography of each IC derived, equivalent current dipole was estimated using Fieldtrip functions [55]. For scalp topographies more suitable for symmetrical bilateral dipoles, two symmetrical dipoles were estimated [56]. To select brain ICs among all types of ICs, EEGLAB plugin ICLabel() was used [57]. The inclusion criteria were 1) 'brain' label probability $>0.7$ and 2) residual variance i.e., var((actual scalp topography)-(theoretical scalp projection from the fitted dipole))/var(actual scalp topography) $<0.15$. Continuous EEG data were segmented into epochs that started at $-250 \mathrm{~ms}$ and end at $750 \mathrm{~ms}$ relative to stimulus onset.

To perform single-trial rejection, for each of $k$ epochs, singletrial power spectral density (PSD) was calculated. There were $\mathrm{m}$ frequency bins between 15 and $35 \mathrm{~Hz}$. Z-scored PSD error from

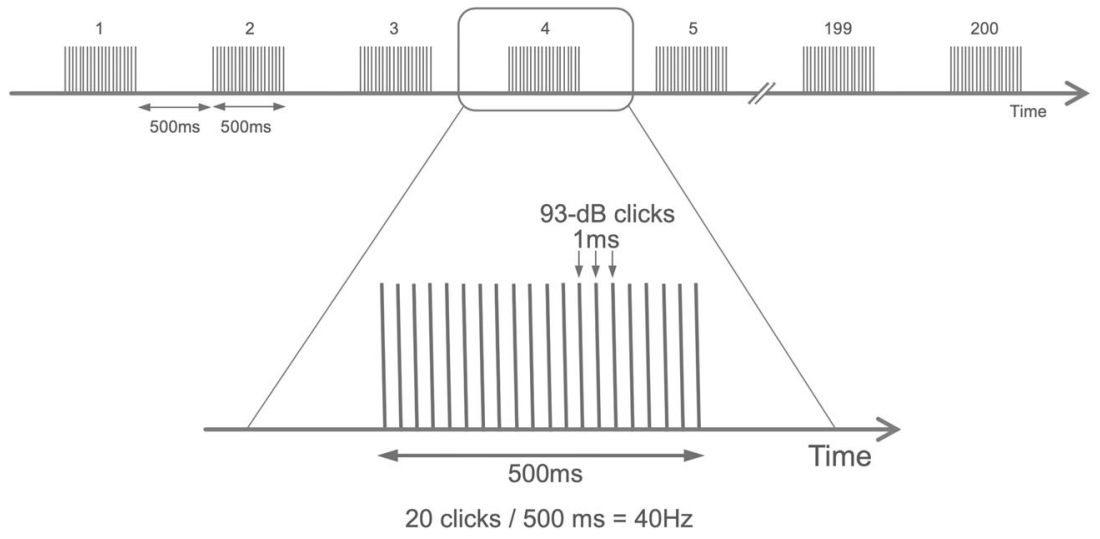

Fig. 1 Gamma-band $(40 \mathrm{~Hz})$ auditory steady-state response paradigm. 
median was calculated as follows.

zscored PSD error $=$ zscore $\left(\frac{1}{m} \sum_{j=1}^{m}\left(\right.\right.$ PSD $\left.\left._{\mathrm{j}, \mathrm{k}}-\operatorname{median}\left(\mathrm{PSD}_{\mathrm{j}}\right)\right)\right)$.

For the distribution of the obtained $k$ Z-scored PSD error values, threshold was applied so that epochs with $Z$-score $>2$ were selected for rejection. The final processed data had a mean of 158.6 trials (SD 18.4).

Dipole density analyses

Inter-trial phase coherence (ITC) $[58,59]$ was calculated for each IC using Short-term Fourier Transform with window size of 64 points ( $256 \mathrm{~ms}$ ) supported by newtimef() included in EEGLAB [46]. ITC is defined as:

$\operatorname{ITC}(f)=\left|\frac{1}{N} \sum_{i=1}^{N} \frac{Z_{i}(f)}{\left|Z_{i}(f)\right|}\right|$,

where $N$ is the number of trials, $Z$ is complex Fourier spectra for frequency $f$. There were 64 frequency bins linearly distributing from 5 to $55 \mathrm{~Hz}$. The maximum ITC value within $0-500 \mathrm{~ms}$ and $35-45 \mathrm{~Hz}$ were picked up and served as representative value for each IC. The wide range of the time window was because we did not have prior knowledge of peak ITC latency for each IC. Out of 9337 brain ICs [3889 Control, 5448 Schizophrenia (Sz)], ICs with peak ITC value $>0.25$ were defined as ASSR responders [11, 15] and selected for further analysis resulting in a remaining $3131 \mathrm{ICs}$ (1402 Control, $1729 \mathrm{Sz}$ ). The cutoff threshold of ITC $>0.25$ was equivalent to 64 and 68 percentiles of peak ITC distributions for the control and schizophrenia groups, respectively.

For the preselected dipoles, their locations in $2 \times 2 \times 2 \mathrm{~mm}$ voxels in the MNI template head model were converted into multivoxel dipole density by applying 3-D Gaussian kernel (full width at half maximum, FWHM $=20 \mathrm{~mm}$ ). Then, the grand-mean whole-brain dipole density data structure was generated for each group separately. These dipole density structure represent probabilistic distribution of IC responders to ASSR. To determine meaningful group differences, we took nonparametric statistical testing approach. First, subtraction was made between the two dipole density structures for each group that generated true group difference in dipole density. For the distribution of the true dipole density difference, we set a cutoff threshold of uncorrected $p<0.01$ (two tailed) to generate an uncorrected significance mask. As a result, seven source clusters were found. Mean dipole density within each of the source cluster was calculated as true group difference in cluster mean, which were to be tested against the subsequently generated surrogate distribution. The same procedure was performed but randomly permuting the group labels to build surrogate group data. Surrogate group difference in dipole density was calculated. The uncorrected significance mask was applied to the surrogate group difference structure to obtain mean dipole density values from the seven source clusters. This procedure was repeated 5,000 times. For each iteration, surrogate group differences of the mean dipole densities within the significance masks across the groups was calculated. Across the seven significance masks, the maximum statistics obtained was stored. Thus the distribution of 5,000 surrogate maximum statistics was constructed. Each of the true group difference values in cluster mean was tested against 95 percentile of the distribution of the surrogate group difference in cluster mean. This procedure provides multiple comparison correction at one-tail $p<0.05$. As a result, six out of seven clusters survived the statistical test with multiple comparison correction using strong familywise error rate (FWER) control. These processes determine source clusters that showed statistically significant group differences in IC dipole density that showed dominant response to ASSR. For the significant six source clusters, their anatomical locations were determined by using MNI-Talairach Converter 1.09 http:// sprout022.sprout.yale.edu/mni2tal/mni2tal.html [60].

To obtain time-frequency decomposed ITC for post hoc analyses, a spherical inclusion mask was set for the 6 source clusters. The radius of the sphere was set to be $17 \mathrm{~mm}$ from following calculation: given FWHM $=20 \mathrm{~mm}$, Gaussian $\sigma$ is 8.5 $\mathrm{mm}$, with which we applied truncation at $2 \sigma$. ICs whose ITC in the gamma-band rage showed peak value $>0.25$ and those that are included within the sphere were included for calculating group difference in ITC. For testing the group difference, a time window of $0-500 \mathrm{~ms}$ and a frequency window of $35-45 \mathrm{~Hz}$ were set as time-frequency window of interest, and average value within the window was calculated. Two-sample $t$ test was used to test the group difference, and false discovery rate (FDR) [61] was used to control inflation of Type I error for testing the six source clusters $(p<0.05$, one tailed). In addition, we also calculated eventrelated spectral perturbation (ERSP) and performed the same analysis procedures for supplementary comparisons of ERSP in the clusters between healthy comparison subjects and schizophrenia patients.

To test the effect of medication, we applied the same analysis described above to the subset of patients $(N=35)$. The same criteria were applied, and seven source clusters were found. The same permutation test was applied to test whether any of these source clusters would survive statistical tests with multiple comparison correction using strong FWER control at one-tail $p<$ 0.05 .

\section{Correlation analyses}

We calculated Pearson correlation coefficients of peak ITC in $40-\mathrm{Hz}$ ASSR at each estimated location with clinical symptoms, neurocognitive function and functional outcomes in schizophrenia patients as an exploratory analysis; statistical significance was set at $p<0.05$. Those equivalent current dipoles that were localized within $2 \sigma(17 \mathrm{~mm}$, given Gaussian distribution with $\mathrm{FWHM}=$ $20 \mathrm{~mm}$ ) from centroids of source clusters were included for the analysis.

\section{RESULTS}

Comparison of source dipole density of gamma-band ASSR (ITC $>0.25$ ) between healthy comparison subjects and schizophrenia patients

Dipole density plots of ICs showing significant gamma synchronization in healthy comparison subjects and schizophrenia patients are seen in Fig. 2. In the healthy comparison group, source clusters were found in the right superior and middle temporal, orbitofrontal, and bilateral sensorimotor cortices. In the schizophrenia group, sources were identified in right superior temporal cortex, orbitofrontal, bilateral sensorimotor cortices, and middle cingulate cortex. These single-group results represent 99th-percentile thresholded dipole density for each group, and as such they are reported only to provide descriptive statistics.

Statistical testing of group differences revealed six source clusters that exhibited a significant reduction of the source density of gamma-band ASSR in schizophrenia patients. The locations of the six source clusters were: the right middle/inferior temporal cortex (cluster volume, $11.6 \mathrm{cc} ; p=0.013$ ), left superior/middle/ inferior temporal cortex (5.6 cc; $p=0.027)$, orbitofrontal cortex (5.7 cc; $p=0.027$ ), left precuneus (2.2 cc; $p=0.042)$, right sensorimotor cortex (8.1 cc; $p=0.020)$ and left superior frontal cortex (1.5 cc; $p=0.0497)$. The results are shown in Fig. 3. MNI coordinates of the centroid of these regions are summarized in Table 1.

As post hoc analyses, we compared ITC at the six source clusters between healthy comparison subjects and schizophrenia patients (Fig. 4). ITC was significantly reduced at the left superior/middle/ inferior temporal cortex, orbitofrontal cortex, left precuneus, right 


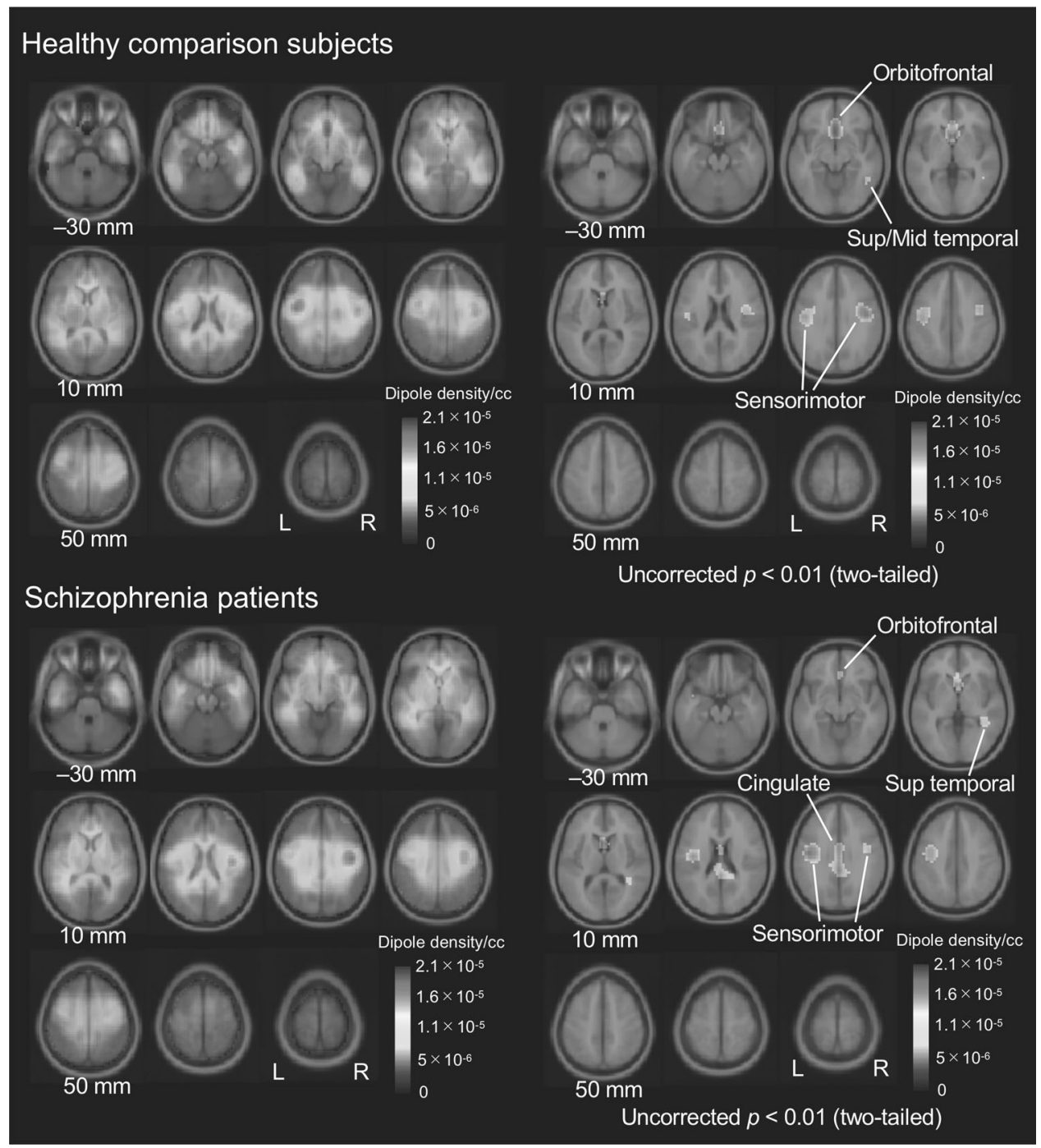

Fig. 2 Source dipole density of gamma-band ASSR (ITC $>\mathbf{0 . 2 5}$ ) in healthy comparison subjects and schizophrenia patients. ASSR auditory steady-state response, ITC inter-trial phase coherence.

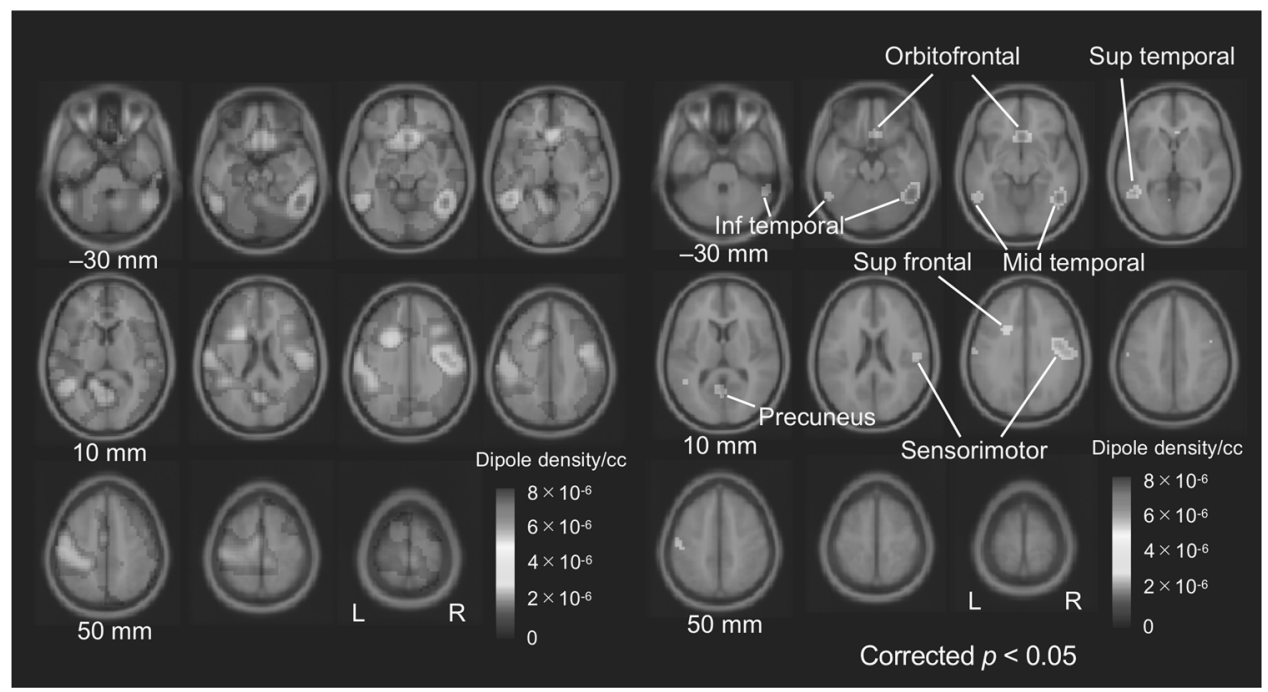

Fig. 3 Difference of source dipole density of gamma-band ASSR (ITC $>0.25$ ) between the groups [(dipole density in healthy comparison subjects) - (dipole density in schizophrenia patients)]. ASSR auditory steady-state response, ITC inter-trial phase coherence. 
Table 1. Six estimated cortical sources in which dipole density of gamma-band ASSR (ITC > 0.25) is significantly reduced in schizophrenia patients compared to healthy comparison subjects.

\begin{tabular}{|c|c|c|c|c|c|}
\hline \multirow[t]{2}{*}{ Brain region } & \multicolumn{3}{|c|}{ Coordinates } & \multirow[t]{2}{*}{ Cluster size } & \multirow[t]{2}{*}{$p$} \\
\hline & $x$ & $y$ & $z$ & & \\
\hline $\begin{array}{l}\text { Temporal cortex, right } \\
\text { Inferior }\end{array}$ & 50 & -52 & -20 & 11.6 & 0.013 \\
\hline $\begin{array}{l}\text { Temporal cortex, left } \\
\text { Superior } \\
\text { Middle } \\
\text { Inferior }\end{array}$ & -52 & -54 & -6 & 5.6 & 0.027 \\
\hline Orbitofrontal cortex & 2 & 20 & -10 & 5.7 & 0.027 \\
\hline Precuneus, left & -4 & -62 & 10 & 2.2 & 0.042 \\
\hline Sensorimotor cortex, right & 48 & -12 & 30 & 8.1 & 0.020 \\
\hline Superior frontal cortex, left & -22 & 12 & 30 & 1.5 & 0.0497 \\
\hline
\end{tabular}

$p$ values are corrected with strong familywise error rate (FWER) control.

ASSR auditory steady-state response, ITC inter-trial phase coherence.

sensorimotor cortex, and left superior frontal cortex, but not at the right middle/inferior temporal cortex in schizophrenia patients compared to healthy comparison subjects. In the additional analysis using ERSP, there is no significant group difference of ERSP at the six source clusters (Supplementary Fig. 1).

We tested the group differences only in the patients who were not prescribed antipsychotics $(N=35)$. Dipole density of these patients that consists of ICs with dominant gamma synchronization (peak ITC $>0.25$ ) is shown in Supplementary Fig. 2. Applying $p<0.01$ threshold (two tailed, uncorrected) found regions including orbitofrontal, bilateral sensorimotor cortex and middle cingulate cortex. The same threshold applied to the group differences revealed seven source clusters (the right middle/ inferior temporal cortex, left superior/middle/inferior cortex, orbitofrontal cortex, left angular gyrus, left sensorimotor cortex; Supplementary Table 2 and Supplementary Fig. 3) that exhibited a reduction of the source density of gamma-band ASSR in schizophrenia patients who were not prescribed antipsychotics $(N=35)$. However, none of these source clusters survived permutation test (all $p>0.05$, after multiple comparison correction using strong FWER control).

Correlations of ITC in gamma-band ASSR with clinical symptoms, cognitive function and functional outcomes in schizophrenia patients

We performed correlation analyses among ITC in 40-Hz ASSR in each estimated location, clinical symptoms, neurocognitive function and functional outcomes in schizophrenia patients as an exploratory analysis. WRAT, LN Span, LN Sequencing, and CVLT scores were significantly lower in schizophrenia patients compared to healthy comparison subjects (Supplementary Table 3). ITC in gamma-band ASSR in right temporal cortex was significantly correlated with SAPS total scores $(r=-0.16$, $p=0.02)$, LN Span scores $(r=0.16, p=0.01)$ and LN Sequencing scores $(r=0.13, p=0.046)$; that in left temporal cortex was significantly correlated with SANS total scores $(r=-0.15, p=$ $0.04)$, WRAT scores $(r=0.17, p=0.01)$, LN sequencing scores $(r=0.17, p=0.02)$ and CVLT scores $(r=0.14, p=0.04)$; that in orbitofrontal cortex was significantly correlated with SANS total scores $(r=-0.13, p=0.04)$; that in left superior frontal cortex was significantly correlated with SANS total scores $(r=-0.15$, $p=0.04)$, CVLT scores $(r=0.17, p=0.02)$ and SOF scores $(r=$ $0.21, p=0.003$ ) in schizophrenia patients (Supplementary Table 4). There is no other significant correlation of ITC in gamma-band ASSR with clinical symptoms, cognitive function or functional outcomes in schizophrenia patients.

\section{DISCUSSION}

In this study, a distributed network of 4 independent cortical ASSR sources was identified in both healthy comparison subjects and schizophrenia patients. In both groups, primary contributing ASSR sources were identified in the right superior temporal cortex and the orbitofrontal cortex, with other contributions including bilateral sensorimotor cortices. Schizophrenia patients showed significantly reduced source dipole density of gamma-band ASSR (ITC > 0.25) in the right middle/inferior temporal cortex, left superior/middle/inferior temporal cortex, orbitofrontal, left precuneus, right sensorimotor cortex, and left superior frontal cortex. ITC was significantly reduced at the left superior/middle/inferior temporal cortex, orbitofrontal cortex, left precuneus, right sensorimotor cortex, and left superior frontal cortex, but not at the right middle/inferior temporal cortex in schizophrenia patients in the post hoc analyses. An exploratory correlation analyses showed that ITC in gamma-band ASSR in bilateral temporal cortices was correlated with positive and negative symptoms and cognitive functions, that in orbitofrontal was correlated with negative symptoms and that in left superior frontal cortex was correlated with negative symptoms, cognitive functions and functional outcomes in schizophrenia patients. These findings clarify the schizophrenia-related basis for gamma phase deficits, an important potential biomarker of the regional cortical dysfunction in schizophrenia.

Results of the present study applied a novel computational approach, to noninvasive scalp recordings. These results were remarkably consistent with findings of cortical surface levels of gamma-band ASSR using invasive ECoG recordings in humans found by Tada et al., who identified prominent gamma-band ASSR responses at superior, middle and inferior temporal cortices, sensorimotor area, as well as prefrontal gyri [40] in patients with treatment-refractory epilepsy. The current study, using novel and noninvasive EEG dipole density analyses fills a critical gap between many previous studies using scalp level EEG [6-16] and the study of Tada et al. [40] using more invasive ECoG, and provides better convergence with translational animal model studies [38].

Our data suggest that the right superior temporal cortex, including the primary auditory cortex (A1), contributes to gammaband ASSR generation in both healthy subjects and in schizophrenia patients. The left superior temporal cortex showed abnormal reduction in the probabilistic density of dominant ASSR in schizophrenia patients alone. We hypothesize that normal auditory information processing may propagate across bilateral middle and inferior temporal cortices in healthy subjects but not in schizophrenia patients. This model is consistent with findings from previous studies of early illness cohorts, where progressive volume reduction within the left superior temporal gyrus was seen in ultrahigh risk for psychosis and in first-episode schizophrenia subjects [62-64]. Local neural capacity to generate gamma-band ASSR centered at the left superior temporal gyrus may be impaired after the onset of psychosis. Indeed, Hirano et al. [9, 65] demonstrated that reduced left hemisphere gamma-band ASSR phase locking in schizophrenia patients was positively correlated with gray matter volume of the left primary auditory cortex. They also showed that significantly increased non-phase locked gamma power (i.e., "induced" activity) during the $40-\mathrm{Hz}$ stimulation was positively correlated with auditory hallucination symptoms [9, 65]. Such a left dominant asymmetry abnormality was similarly observed in the current study. Although we found a correlation between positive symptom and gamma-band ASSR in the right temporal cortex but not in the left temporal cortex, the difference between the region that covered broad area including secondary 
Healthy comparison subjects

Temporal cortex, right (middle/inferior)
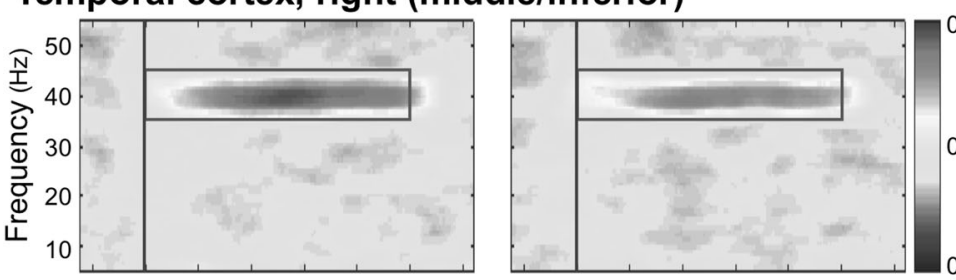

Temporal cortex, left (superior/middle/inferior)

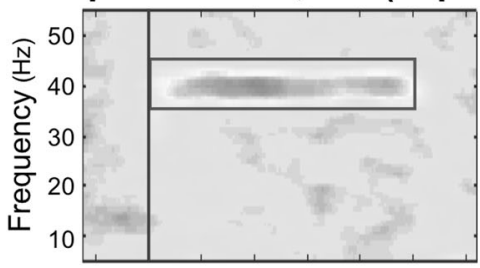

Orbitofrontal cortex

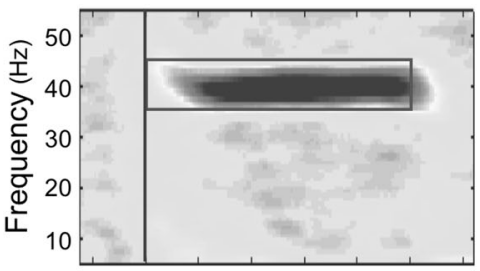

Precuneus, left

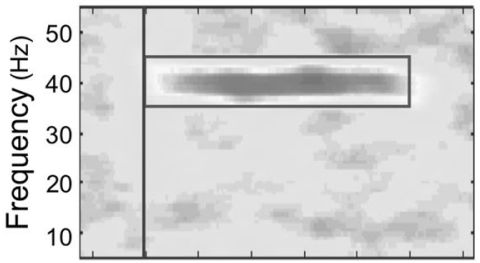

Sensorimotor cortex, right

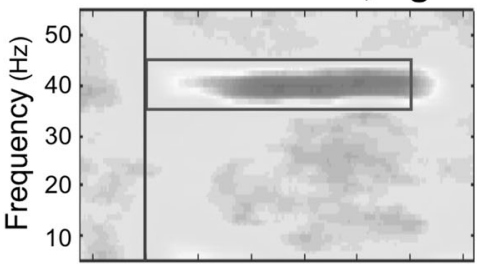

Superior frontal cortex, left
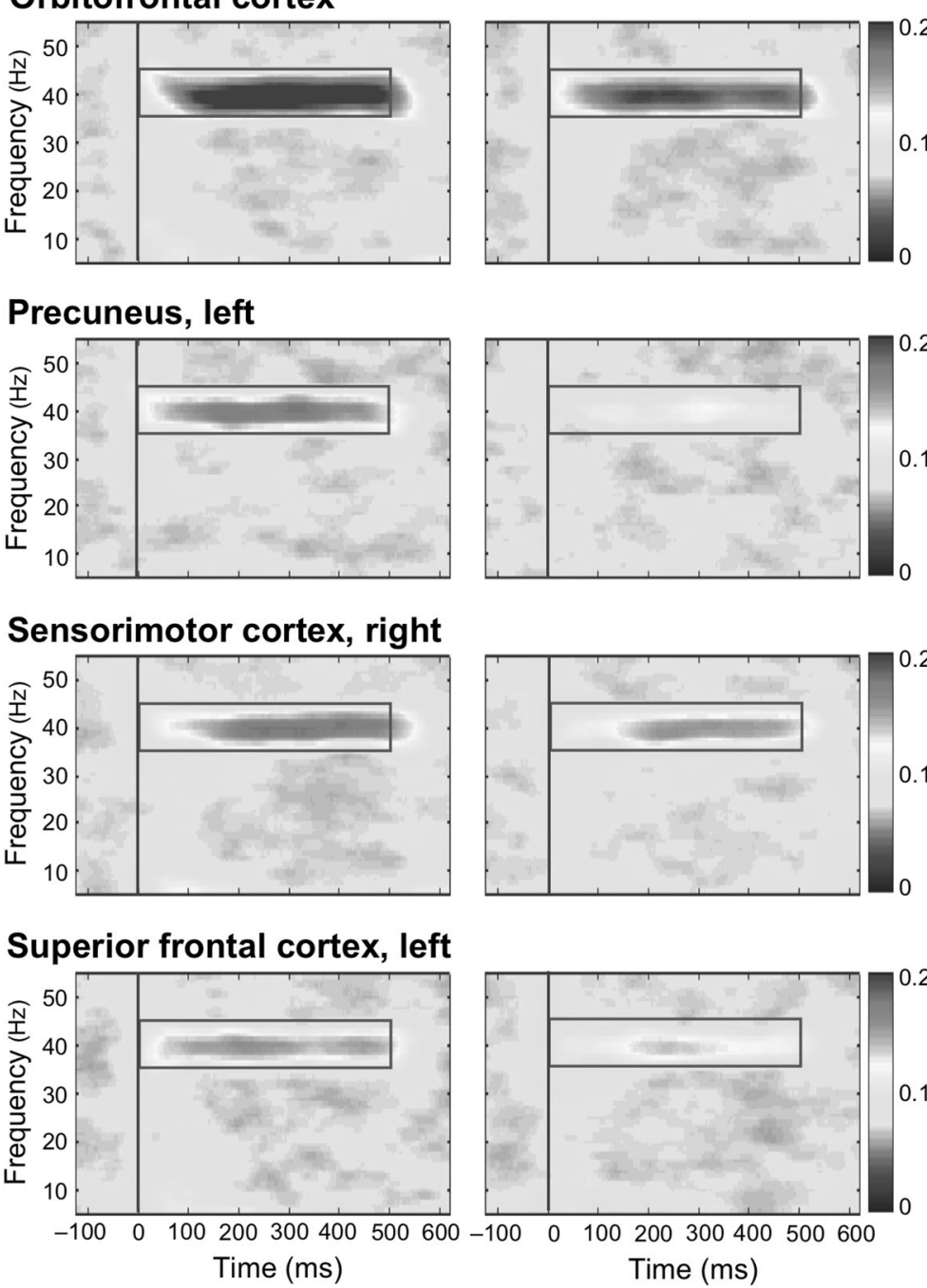
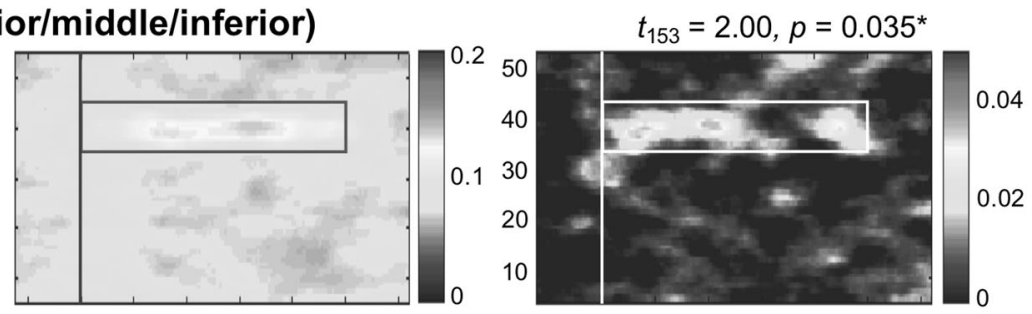

\section{Difference}

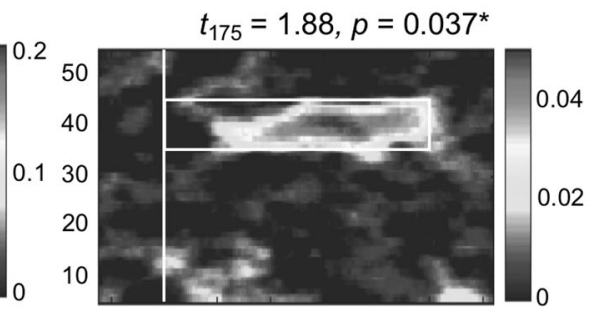

$t_{175}=1.88, p=0.037^{\star}$
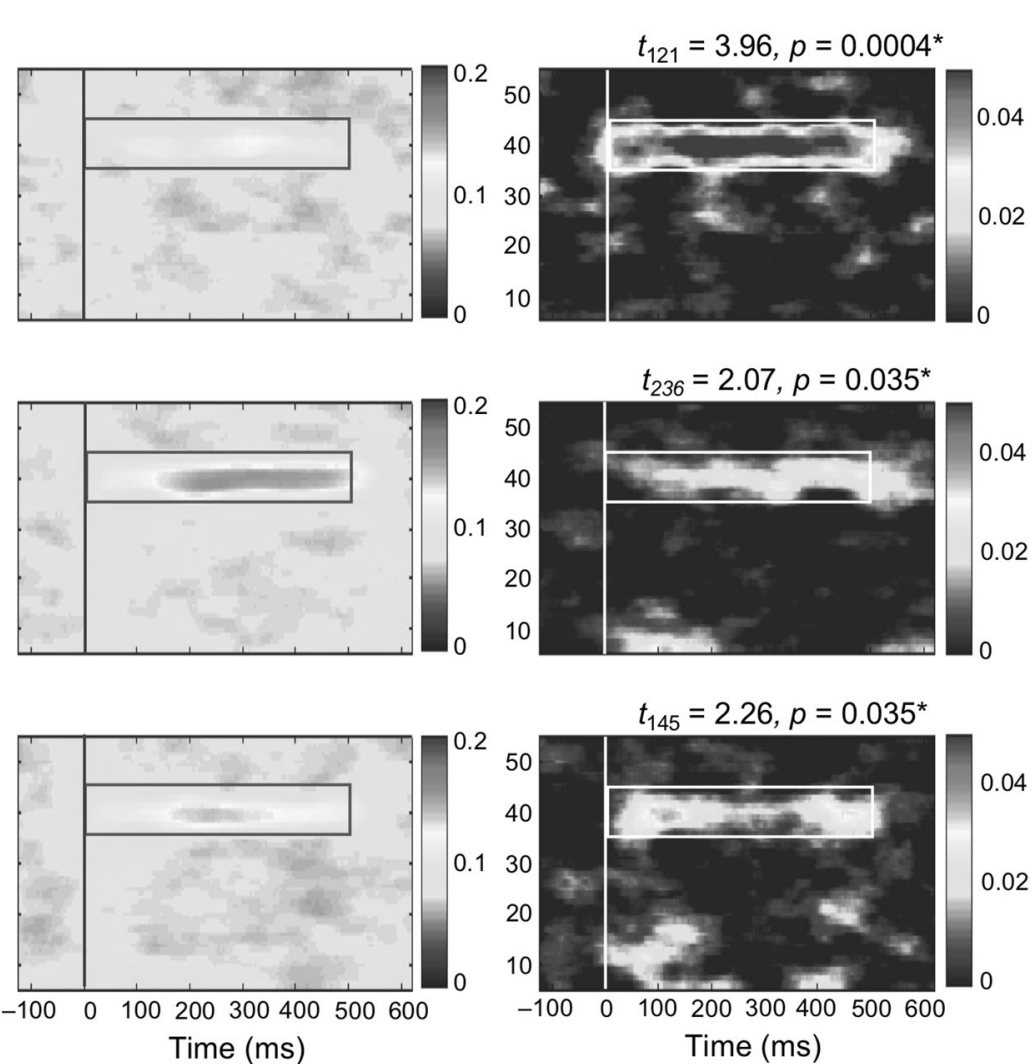

Fig. 4 The grand average time-frequency maps for ITC at six source clusters. Six source clusters in which dipole density of gamma-band ASSR (ITC > 0.25) is significantly reduced in schizophrenia patients compared to healthy comparison subjects (Table 1). Asterisks indicate statistically significance at $p<0.05$ (FDR correction applied, one tail) in comparisons of ITC between healthy comparison subjects and schizophrenia patients [(ITC in healthy comparison subjects) - (ITC in schizophrenia patients)] at each source cluster. A time window of $0-500 \mathrm{~ms}$ and a frequency window of $35-45 \mathrm{~Hz}$ were set as time-frequency window of interest, and average value within the window was calculated. ITC inter-trial phase coherence, FDR false discovery rate. 
auditory cortex and only region that covered primary auditory cortex might affect the different results.

Given the relative "simplicity" of the ASSR paradigm, the significant group differences in orbitofrontal and prefrontal cortices were not expected; these regions are usually associated with higher order cognitive functions, rather than a task involving the passive response to simple trains of click stimuli. Nonetheless, this study is not the first to report frontal contributions to ASSR. A recent study of 19 healthy subjects also reported source locations of gamma-band ASSR in the inferior portion of the orbitofrontal cortex and anterior cingulate cortex [39]. Here, we replicated these findings and identified source locations of reduced gamma-band ASSR in the orbitofrontal cortex and anterior cingulate cortex in schizophrenia. Furthermore, we replicated a correlation between reduced gamma-band ASSR in the prefrontal cortex and neurocognitive functioning in schizophrenia patients [66]. These results suggest that the conventional view of the ASSR as a lowlevel perceptual response with exclusive generation by auditory cortex should be reconsidered. On the contrary, our results indicate that gamma-band ASSR may also serve as a productive probe of the functioning of a distributed network that also includes important contributions from frontal cortical sources. The rapid (i.e., usually $<5 \mathrm{~min}$ ) assessment of ASSR combined with low cognitive and effort demands of the task lends itself to more widespread use in diagnostic and precision medicine trials among patients with cognitive and motivational impairments that may confound performance in other tasks (cf. [67]).

A previous study showed that in the vibrotactile frequency discrimination task, tactile information, and auditory information can be used cooperatively in the secondary somatosensory and auditory cortices [68]. Although speculative, a similar mechanism may be evoked by ASSR task. Similarly, the sensorimotor area may also have a role in gamma-band ASSR. The precuneus is a critical cortical node of oscillatory activity coordinating cross-regional interactions for visuo-spatial imagery, episodic memory retrieval and self-processing operations $[69,70]$. Given those findings and the location of the precuneus, the precuneus may also contribute to integrate auditory information processing during oscillation task.

\section{Caveats}

First, this is a cross-sectional cohort study of a heterogeneous sample of schizophrenia patients, the majority of whom were receiving complex medication regimens. Testing group difference of source density of gamma-band ASSR between patients who were not prescribed antipsychotics $(N=35)$ and healthy comparison subjects $(N=293)$, significance of original group difference between patients $(N=427)$ and healthy comparison subjects $(N=$ 293) did not remain after permutation test with multiple comparison correction using strong FWER control. Given a previous finding of correlations between global functioning and gamma-band ASSR in schizophrenia patients [14], patients who were prescribed antipsychotics may show lower global functioning and more severe reduction of source density of gamma-band ASSR compared to healthy comparison subjects. Indeed, SOF scores are significantly lower in patients who were prescribed antipsychotics than in those who were not prescribed them $\left(t_{410}=\right.$ $-2.24, p=0.025)$. Second, 40 EEG channels were used for the analyses in this study. Future studies may fruitfully use higher density recordings with at least 64 channels [71], individual MRI data, and digitized scalp sensor locations rather than template head models and reliance on standardized electrode locations for potentially improved accuracy of source dynamics. Third, the schizophrenia patients in this study had a "chronic" illness; results therefore may not generalize to at risk or early illness psychosis patients.

\section{CONCLUSION}

We successfully identified cortical source locations of synchronized gamma frequency oscillations at frontotemporal regions in schizophrenia patients by applying a novel approach using ICA modeling to scalp EEG recordings. Our findings provide evidence that deficits in the generation of gamma-band oscillations in response to $40-\mathrm{Hz}$ stimuli reflect abnormalities across a distributed network of temporal and frontal brain regions in schizophrenia patients. Clarification of the neural mechanisms underlying the networks detected in this study, in both future clinical and animal studies, will strengthen the utility of gamma-band ASSR as a translatable brain marker for clarifying the pathophysiology of neuropsychiatric and neurological diseases and the development of novel therapeutic interventions.

\section{FUNDING AND DISCLOSURE}

This study was supported by JSPS Overseas Research Fellowships (DK), the Sidney R. Baer, Jr. Foundation, and the VISN-22 Mental Illness Research, Education, and Clinical Center. Swartz Center for Computational Neuroscience is supported by generous gift of Swartz Foundation (New York). The funders had no role in the study design, data collection and analysis, publication decision, or paper preparation. The authors declare no competing interests.

\section{ACKNOWLEDGEMENTS}

We gratefully acknowledge all the participants of this study.

\section{AUTHOR CONTRIBUTIONS}

JS, DLB, NRS, and GAL collected the data. DK and MM analyzed the data. DK, MM, YBJ, JLM, KTK, DLB, NRS, and GAL interpreted the results. DK, MM, and GAL designed the study. GAL supervised all aspects of collection, analysis, and interpretation of the data. DK, MM, and GAL wrote the original paper. YBJ, JLM, KTK, JS, DLB, and NRS reviewed and edited the paper. All authors contributed to and approved the final paper.

\section{ADDITIONAL INFORMATION}

Supplementary Information accompanies this paper at (https://doi.org/10.1038/ s41386-020-00806-5).

Publisher's note Springer Nature remains neutral with regard to jurisdictional claims in published maps and institutional affiliations.

\section{REFERENCES}

1. Uhlhaas PJ, Singer W. Abnormal neural oscillations and synchrony in schizophrenia. Nat Rev Neurosci. 2010;11:100-13.

2. Tada M, Kirihara K, Koshiyama D, Fujioka M, Usui K, Uka T, et al. Gamma-band auditory steady-state response as a neurophysiological marker for excitation and inhibition balance: a review for understanding schizophrenia and other neuropsychiatric disorders. Clin EEG Neurosci. 2019;51:234-43.

3. Galambos R, Makeig S, Talmachoff PJ. A 40-Hz auditory potential recorded from the human scalp. Proc Natl Acad Sci USA. 1981;78:2643-7.

4. Galambos R, Makeig S. Physiological studies of central masking in man. I: the effects of noise on the $40-\mathrm{Hz}$ steady-state response. J Acoust Soc Am. 1992;92:2683-90.

5. Makeig S. Auditory event-related dynamics of the EEG spectrum and effects of exposure to tones. Electroencephalogr Clin Neurophysiol. 1993;86:283-93.

6. Kwon JS, O'Donnell BF, Wallenstein GV, Greene RW, Hirayasu Y, Nestor PG, et al. Gamma frequency-range abnormalities to auditory stimulation in schizophrenia. Arch Gen Psychiatry. 1999;56:1001-5.

7. Brenner CA, Sporns O, Lysaker PH, O'Donnell BF. EEG synchronization to modulated auditory tones in schizophrenia, schizoaffective disorder, and schizotypal personality disorder. Am J Psychiatry. 2003;160:2238-40.

8. Hamm JP, Bobilev AM, Hayrynen LK, Hudgens-Haney ME, Oliver WT, Parker DA, et al. Stimulus train duration but not attention moderates gamma-band entrainment abnormalities in schizophrenia. Schizophr Res. 2015;165:97-102. 
9. Hirano Y, Oribe N, Kanba S, Onitsuka T, Nestor PG, Spencer KM. Spontaneous gamma activity in schizophrenia. JAMA Psychiatry. 2015;72:813-21.

10. Kirihara K, Rissling AJ, Swerdlow NR, Braff DL, Light GA. Hierarchical organization of gamma and theta oscillatory dynamics in schizophrenia. Biol Psychiatry. 2012;71:873-80.

11. Light GA, Hsu JL, Hsieh MH, Meyer-Gomes K, Sprock J, Swerdlow NR, et al. Gamma band oscillations reveal neural network cortical coherence dysfunction in schizophrenia patients. Biol Psychiatry. 2006;60:1231-40.

12. Spencer KM, Salisbury DF, Shenton ME, McCarley RW. Gamma-band auditory steady-state responses are impaired in first episode psychosis. Biol Psychiatry. 2008;64:369-75.

13. Tada M, Nagai T, Kirihara K, Koike S, Suga M, Araki T, et al. Differential alterations of auditory gamma oscillatory responses between pre-onset high-risk individuals and first-episode schizophrenia. Cereb Cortex. 2016;26:1027-35.

14. Koshiyama D, Kirihara K, Tada M, Nagai T, Fujioka M, Ichikawa E, et al. Auditory gamma oscillations predict global symptomatic outcome in the early stages of psychosis: a longitudinal investigation. Clin Neurophysiol. 2018;129:2268-75.

15. Koshiyama D, Kirihara K, Tada M, Nagai T, Fujioka M, Ichikawa E, et al. Electrophysiological evidence for abnormal glutamate-GABA association following psychosis onset. Transl Psychiatry. 2018;8:211.

16. Koshiyama D, Kirihara K, Tada M, Nagai T, Fujioka M, Usui K, et al. Gamma-band auditory steady-state response is associated with plasma levels of $\mathrm{d}$-serine in schizophrenia: an exploratory study. Schizophr Res. 2019;208:467-69.

17. Edgar JC, Chen YH, Lanza M, Howell B, Chow VY, Heiken K, et al. Cortical thickness as a contributor to abnormal oscillations in schizophrenia? Neuroimage Clin. 2014;4:122-9.

18. Teale P, Collins D, Maharajh K, Rojas DC, Kronberg E, Reite M. Cortical source estimates of gamma band amplitude and phase are different in schizophrenia. Neuroimage. 2008;42:1481-9.

19. Tsuchimoto R, Kanba S, Hirano S, Oribe N, Ueno T, Hirano $Y$, et al. Reduced high and low frequency gamma synchronization in patients with chronic schizophrenia. Schizophr Res. 2011;133:99-105

20. Vierling-Claassen D, Siekmeier $P$, Stufflebeam S, Kopell N. Modeling GABA alterations in schizophrenia: a link between impaired inhibition and altered gamma and beta range auditory entrainment. J Neurophysiol. 2008;99:2656-71.

21. Wilson TW, Hernandez OO, Asherin RM, Teale PD, Reite ML, Rojas DC. Cortical gamma generators suggest abnormal auditory circuitry in early-onset psychosis. Cereb Cortex. 2008;18:371-8.

22. Thune $\mathrm{H}$, Recasens $M$, Uhlhaas $\mathrm{PJ}$. The $40-\mathrm{Hz}$ auditory steady-state response in patients with schizophrenia: a meta-analysis. JAMA Psychiatry. 2016;73:1145-53.

23. O'Donnell BF, Hetrick WP, Vohs JL, Krishnan GP, Carroll CA, Shekhar A. Neural synchronization deficits to auditory stimulation in bipolar disorder. Neuroreport. 2004;15:1369-72.

24. Reite M, Teale P, Rojas DC, Reite E, Asherin R, Hernandez O. MEG auditory evoked fields suggest altered structural/functional asymmetry in primary but not sec ondary auditory cortex in bipolar disorder. Bipolar Disord. 2009;11:371-81.

25. Rass O, Krishnan G, Brenner CA, Hetrick WP, Merrill CC, Shekhar A, et al. Auditory steady state response in bipolar disorder: relation to clinical state, cognitive performance, medication status, and substance disorders. Bipolar Disord. 2010;12:793-803.

26. Oda Y, Onitsuka T, Tsuchimoto R, Hirano S, Oribe N, Ueno T, et al. Gamma band neural synchronization deficits for auditory steady state responses in bipolar disorder patients. PLOS ONE. 2012;7:e39955.

27. Isomura S, Onitsuka T, Tsuchimoto R, Nakamura I, Hirano S, Oda Y, et al. Differentiation between major depressive disorder and bipolar disorder by auditory steady-state responses. J Affect Disord. 2016;190:800-6.

28. Edgar JC, Fisk CLT, Liu S, Pandey J, Herrington JD, Schultz RT, et al. Translating adult electrophysiology findings to younger patient populations: difficulty measuring $40-\mathrm{Hz}$ auditory steady-state responses in typically developing children and children with autism spectrum disorder. Dev Neurosci. 2016;38:1-14.

29. Larsen KM, Pellegrino G, Birknow MR, Kjaer TN, Baare WFC, Didriksen M, et al. 22 11.2 deletion syndrome is associated with impaired auditory steady-state gamma response. Schizophr Bull. 2018;44:388-97.

30. Cardin JA, Carlen M, Meletis K, Knoblich U, Zhang F, Deisseroth K, et al. Driving fast-spiking cells induces gamma rhythm and controls sensory responses. Nature. 2009;459:663-7.

31. Sohal VS, Zhang F, Yizhar $O$, Deisseroth K. Parvalbumin neurons and gamma rhythms enhance cortical circuit performance. Nature. 2009;459:698-702.

32. Akbarian S, Huang HS. Molecular and cellular mechanisms of altered GAD1/ GAD67 expression in schizophrenia and related disorders. Brain Res Rev. 2006;52:293-304.

33. Eyles DW, McGrath JJ, Reynolds GP. Neuronal calcium-binding proteins and schizophrenia. Schizophr Res. 2002;57:27-34

34. Carlen M, Meletis K, Siegle JH, Cardin JA, Futai K, Vierling-Claassen D, et al. A critical role for NMDA receptors in parvalbumin interneurons for gamma rhythm induction and behavior. Mol Psychiatry. 2012;17:537-48.
35. Nakao K, Nakazawa K. Brain state-dependent abnormal LFP activity in the auditory cortex of a schizophrenia mouse model. Front Neurosci. 2014;8:168.

36. Fisahn A, Neddens J, Yan L, Buonanno A. Neuregulin-1 modulates hippocampal gamma oscillations: implications for schizophrenia. Cereb Cortex. 2009;19: 612-8.

37. Gonzalez-Burgos G, Cho RY, Lewis DA. Alterations in cortical network oscillations and parvalbumin neurons in schizophrenia. Biol Psychiatry. 2015;77:1031-40.

38. Kozono N, Honda S, Tada M, Kirihara K, Zhao Z, Jinde S, et al. Auditory steady state response; nature and utility as a translational science tool. Sci Rep. 2019;9:8454.

39. Farahani ED, Goossens T, Wouters J, van Wieringen A. Spatiotemporal reconstruction of auditory steady-state responses to acoustic amplitude modulations: potential sources beyond the auditory pathway. Neuroimage. 2017;148:240-53.

40. Tada M, Kirihara K, Ishishita Y, Takasago M, Kunii N, Uka T, et al. Global and parallel cortical processing of auditory gamma oscillatory responses in humans. 2019; Cell-Reports-D-19-02197. https://ssrn.com/abstract=3417938 or https://doi. org/10.2139/ssrn.3417938.

41. Bell AJ, Sejnowski TJ. An information-maximization approach to blind separation and blind deconvolution. Neural Comput. 1995;7:1129-59.

42. Onton J, Makeig S. Information-based modeling of event-related brain dynamics. Prog Brain Res. 2006;159:99-120.

43. Fausti SA, Wilmington DJ, Helt PV, Helt WJ, Konrad-Martin D. Hearing health and care: the need for improved hearing loss prevention and hearing conservation practices. J Rehabil Res Dev. 2005:42:45-62.

44. Oostenveld $R$, Praamstra $P$. The five percent electrode system for high-resolution EEG and ERP measurements. Clin Neurophysiol. 2001;112:713-9.

45. Collins DL, Neelin P, Peters TM, Evans AC. Automatic 3D intersubject registration of MR volumetric data in standardized Talairach space. J Comput Assist Tomogr. 1994;18:192-205.

46. Delorme A, Makeig S. EEGLAB: an open source toolbox for analysis of single-trial EEG dynamics including independent component analysis. J Neurosci Methods. 2004;134:9-21.

47. Chang $\mathrm{CY}$, Hsu SH, Pion-Tonachini L, Jung TP. Evaluation of artifact subspace reconstruction for automatic EEG artifact removal. Conf Proc IEEE Eng Med Biol Soc. 2018;2018:1242-45.

48. Blum S, Jacobsen NSJ, Bleichner MG, Debener S. A riemannian modification of artifact subspace reconstruction for EEG artifact handling. Front Hum Neurosci. 2019;13:141.

49. Chang CY, Hsu SH, Pion-Tonachini L, Jung TP. Evaluation of artifact subspace reconstruction for automatic artifact components removal in multi-channel EEG recordings. IEEE Trans Biomed Eng. 2020;67:1114-21.

50. Gabard-Durnam LJ, Mendez Leal AS, Wilkinson CL, Levin AR. The harvard automated processing pipeline for electroencephalography (HAPPE): standardized processing software for developmental and high-artifact data. Front Neurosci. 2018;12:97.

51. Mullen TR, Kothe CA, Chi YM, Ojeda A, Kerth T, Makeig $S$, et al. Real-time neuroimaging and cognitive monitoring using wearable dry EEG. IEEE Trans Biomed Eng. 2015;62:2553-67.

52. Kothe CA, Makeig S. BCILAB: a platform for brain-computer interface development. J Neural Eng. 2013;10:056014.

53. Palmer J, Kreutz-delgado K, Makeig S. AMICA: an adaptive mixture of independent component analyzers with shared components; 2016. http://citeseerx.ist. psu.edu/viewdoc/summary?doi=10.1.1.295.1351.

54. Delorme A, Palmer J, Onton J, Oostenveld R, Makeig S. Independent EEG sources are dipolar. PLoS ONE. 2012;7:e30135.

55. Oostenveld R, Fries $P$, Maris $E$, Schoffelen JM. FieldTrip: open source software for advanced analysis of MEG, EEG, and invasive electrophysiological data. Comput Intell Neurosci. 2011;2011:156869.

56. Piazza C, Miyakoshi M, Akalin-Acar Z, Cantiani C, Reni G, Bianchi AM in XIV mediterranean conference on medical and biological engineering and computing. Springer International Publishing; 2016. p. 105-09.

57. Pion-Tonachini L, Kreutz-Delgado K, Makeig S. ICLabel: An automated electroencephalographic independent component classifier, dataset, and website. Neuroimage. 2019;198:181-97.

58. Keitel C, Keitel A, Benwell CSY, Daube C, Thut G, Gross J. Stimulus-driven brain rhythms within the alpha band: the attentional-modulation conundrum. J Neurosci. 2019;39:3119-29.

59. Tallon-Baudry C, Bertrand O, Delpuech C, Pernier J. Stimulus specificity of phaselocked and non-phase-locked $40 \mathrm{~Hz}$ visual responses in human. J Neurosci. 1996;16:4240-9.

60. Lacadie CM, Fulbright RK, Rajeevan N, Constable RT, Papademetris X. More accurate Talairach coordinates for neuroimaging using non-linear registration. Neuroimage. 2008:42:717-25.

61. Benjamini $Y$, Hochberg Y. Controlling the false discovery rate: a practical and powerful approach to multiple testing. J R Statist Soc B. 1995;57:289-300. 
62. Takahashi T, Wood SJ, Yung AR, Walterfang M, Phillips LJ, Soulsby B, et al. Superior temporal gyrus volume in antipsychotic-naive people at risk of psychosis. Br J Psychiatry. 2010;196:206-11.

63. Kasai K, Shenton ME, Salisbury DF, Hirayasu Y, Lee CU, Ciszewski AA, et al. Progressive decrease of left superior temporal gyrus gray matter volume in patients with first-episode schizophrenia. Am J Psychiatry. 2003;160:156-64.

64. Kasai K, Shenton ME, Salisbury DF, Hirayasu Y, Onitsuka T, Spencer MH, et al. Progressive decrease of left Heschl gyrus and planum temporale gray matter volume in first-episode schizophrenia: a longitudinal magnetic resonance imaging study. Arch Gen Psychiatry. 2003;60:766-75.

65. Hirano Y, Oribe N, Onitsuka T, Kanba S, Nestor PG, Hosokawa T, et al. Auditory cortex volume and gamma oscillation abnormalities in schizophrenia. Clin EEG Neurosci. 2020;51:244-51.

66. Senkowski D, Gallinat J. Dysfunctional prefrontal gamma-band oscillations reflect working memory and other cognitive deficits in schizophrenia. Biol Psychiatry. 2015;77:1010-9.
67. Bismark AW, Thomas ML, Tarasenko M, Shiluk AL, Rackelmann SY, Young JW, et al. Relationship between effortful motivation and neurocognition in schizophrenia. Schizophr Res. 2018;193:69-76.

68. Iguchi $Y$, Hoshi $Y$, Nemoto $M$, Taira $M$, Hashimoto I. Co-activation of the secondary somatosensory and auditory cortices facilitates frequency discrimination of vibrotactile stimuli. Neuroscience. 2007;148:461-72.

69. Cavanna AE, Trimble MR. The precuneus: a review of its functional anatomy and behavioural correlates. Brain. 2006;129:564-83.

70. Hebscher M, Meltzer JA, Gilboa A. A causal role for the precuneus in networkwide theta and gamma oscillatory activity during complex memory retrieval. Elife. 2019;8:e43114.

71. Light GA, Swerdlow NR. Selection criteria for neurophysiologic biomarkers to accelerate the pace of CNS therapeutic development. Neuropsychopharmacology. 2020;45:237-8. 